\title{
Experimental research in topical psoriasis therapy (Review)
}

\author{
DIANA ANA-MARIA NIȚESCU ${ }^{*}$, ALINA MUȘETESCU ${ }^{2,3}$, MARIA NIȚESCU $^{4 *}$, \\ MONICA COSTESCU ${ }^{2,5}$ and OANA-ANDREIA COMAN ${ }^{1 *}$
}

\author{
${ }^{1}$ Department of Pharmacology and Pharmacotherapy, Faculty of Medicine, \\ 'Carol Davila' University of Medicine and Pharmacy, 050474 Bucharest; ${ }^{2}$ Department of Dermatovenereology, \\ 'Dr. Victor Babes' Clinical Hospital for Infectious and Tropical Diseases, 030303 Bucharest; \\ ${ }^{3}$ Department of Dermatovenereology, Faculty of Medicine, 'Titu Maiorescu' University, 040051 Bucharest; \\ ${ }^{4}$ Department of Hygiene and Medical Ecology, Faculty of Medicine, ${ }^{5}$ Department of Dermatovenereology, \\ Faculty of Dental Medicine, 'Carol Davila' University of Medicine and Pharmacy, 050474 Bucharest, Romania
}

Received January 5, 2021; Accepted February 4, 2021

DOI: $10.3892 / \mathrm{etm} .2021 .10403$

\begin{abstract}
Psoriasis, one of the most prevalent inflammatory diseases in dermatologic pathology, remains a challenge in regards to the therapeutic approach. Topical therapy for psoriasis is a current trending subject as it implies good compliance for the patient, few adverse systemic reactions and a targeted effect. Numerous substances are now being tested, from natural to synthetic compounds and already known substances in improved formulas such as vesicular systems. The aim of this article was to conduct a literature review regarding the topical therapy of psoriasis in animal models, between June, 27, 2019 and July 9, 2020. For this article, the authors conducted extensive research in PubMed with the following keywords: Psoriasis AND (topical OR local) and (therapy OR treatment) AND (mice OR rats). The main new studied substances included lycopene, sodium butyrate, salvianolic acid B, small interfering RNAs (siRNAs) in ionic liquids, albendazole, phosphodiesterase inhibitors, biomimetic reconstituted highdensity lipoprotein nanocarrier gel containing microRNA (miRNA)-210 antisense, thymoquinone in ethosomal vesicle, Sea buckthorn oil (Hippophae rhamnoides), nitidine chloride, Melissa officinalis spp. Altissima extract and [1-(4-chloro-3nitrobenzenesulfonyl)-1H-indol-3-yl]-methanol (CIM). New formulas of already known anti-psoriasis substances such as: Cyclosporine, methotrexate, calcipotriol, tazarotene, protein kinase p38 and integrin $\alpha 5 \beta 1$ as a target, are also reviewed. Recent research in topical psoriasis underlines the importance of animal experimental research in dermatology, providing
\end{abstract}

Correspondence to: Dr Alina Mușetescu, Department of Dermatovenereology, Faculty of Medicine, 'Titu Maiorescu' University, 22 Dambovnicului Street, 040051 Bucharest, Romania

E-mail: alina.musetescu@gmail.com

${ }^{*}$ Contributed equally

Key words: psoriasis, topical, treatment, mice, imiquimod a starting point for developing new therapeutic approaches in one of the most frequently diagnosed chronic dermatologic diseases. Vesicular systems are now providing the best vehicle for topical therapy, thus easing the action of the active substances at their target sites.

\section{Contents}

1. Introduction

2. Research methods

3. Summary and discussion of the new topical treatment strategies

4. Discussion

5. Conclusions

\section{Introduction}

Psoriasis represents a chronic, incurable, immune-mediated disease, being a combination between polygenic predisposition and external triggers (trauma, microorganism, drugs) or internal triggers (stress) (1). Psoriasis is one of the most prevalent dermatological diseases and a continuous challenge in regards to therapeutic approach.

The key in initiating the characteristic lesions of psoriasis is dendritic cell stimulation by Toll-like receptors (TLRs) with interferon (IFN $\alpha$ and IFN $\beta$ ) production, followed by regional ganglia migration and tumor necrosis factor (TNF $\alpha$, interleukin (IL)-23 and IL-12 production. The latter (IL-23 and IL-12) stimulate LTh17 and LTh1 subtype differentiation. In the steady phase, adaptative immune system is activated with Th-17 cytokine secretion (IL-17, IL-21, IL-22) and dermal proliferation. Therefore, the TNF $\alpha / \mathrm{IL}-23 / \mathrm{Th}-17$ axis is characteristic for psoriasis vulgaris and psoriatic arthritis (2). Intracellular activated kinases through this way include: Extracellular signal-regulated kinase (ERK), p38 MAPK (mitogen-activated protein kinases), transforming growth factor (TGF)- $\beta$-activated kinase 1 (TAK1), IкB kinase (IKK), and glycogen synthase kinase $3 \beta$ (GSK-3 $\beta$ ). These kinases enable nuclear factor (NF)- $\mathrm{\kappa B}, \mathrm{AP}-1$, and $\mathrm{C} / \mathrm{EBP}$ transcription 
of pro-inflammatory cytokines, chemokines, and antimicrobial peptides (3-6).

Currently, along with evolving systemic therapy, biological treatment has revolutionized severe psoriasis management. Topical treatment is especially recommended for localized and moderate forms of psoriasis and aims to decrease the severity of hyperkeratosis (preparations with tar, dioxyanthranol, salicylic acid) and decrease keratinocyte proliferation (topical retinoids, vitamin D3 analogues). Dermatocorticoids are first-line active agents in the topical treatment of psoriasis with important local side effects such as atrophy, hypopigmentation, and superinfections.

The experimental models used to evaluate psoriasis changes in laboratory animals are mainly the imiquimod (IMQ) mouse model, the mouse tail model, and the carrageenan and TPA (12-O-tetradecanoylphorbol-13-acetate) inflammation induction models.

The present trend is to discover either new effective or already approved substances conditioned in superior formulas in regards to absorption and efficacy and new mechanisms with anti-psoriasis potential.

\section{Research methods}

The aim of this article was to conduct a literature review regarding research on the topical therapy of psoriasis in animal models, between June, 27, 2019 and July 9, 2020.

For this article, the authors conducted extensive research in PubMed with the following keywords: Psoriasis AND (topical OR local) AND (therapy OR treatment) with publication dates January 1, 1995 to July 15, 2020 for search 1 and also psoriasis AND (topical OR local) AND (therapy OR treatment) AND (mice OR rats) for search 2.

The first search was made in order to assess the scientific interest in the topic. The second search had the following steps: Introduction of words and limits in PubMed with first step exclusion criteria for articles that did not refer to a potential substance for topical therapy, formulas from traditional medicine that did not specify the active ingredient (7-17). The second step exclusion criterion was if the substance of interest was not administered topically. The search was performed by two authors in the PubMed database and the results were mediated.

The data extracted from each article referred to newly tested substance or known substance with a new pharmaceutical form (dosage, pharmaceutical form, concentration), the experimental model used and the mechanism of action involved.

\section{Summary and discussion of the new topical treatment strategies}

Interest in topical psoriasis therapy has increased over time. Regarding the number of articles between 1951 and 2020, the first keywords were used (see search 1).

The total number of articles was 6,052 (4,572 between 1995 and 2020) until July 9, 2020. In the last 25 years, the interest has increased with a peak in 2018 and a slight decrease over the last 2 years (Fig. 1). The trendline is at an average of 5 years. In the last year (June 27, 2019 to July 9, 2020),

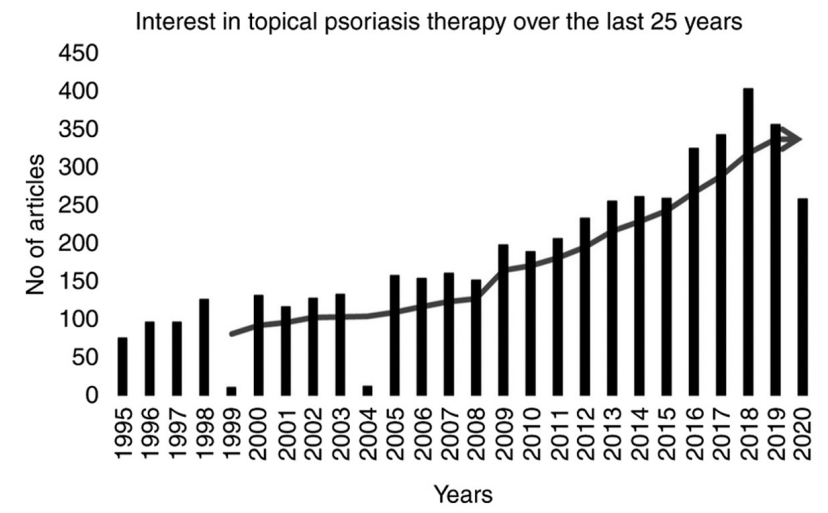

Figure 1. Interest in topical anti-psoriasis therapy over the last 25 years.

41 articles were found and 23 were deemed articles of interest (Fig. 2) and are summarized as follows in Tables I and II.

In a study by Shih et al lycopene, a terpenoid carotenoid, was administered orally at a dose of 0.06 and $0.12 \mathrm{mg} / \mathrm{kg} /$ day and topically at a dose of 0.06 and $0.12 \mathrm{mg} / \mathrm{ml}$ of ointment in the imiquimod (IMQ) model. The results revealed a dosedependent decrease in the Psoriasis Area Severity Index (PASI), epidermal hyperplasia and cell adhesion on HaCaT cultures stimulated by TNF $\alpha$, demonstrating the anti-psoriasis effect (18) (Table II).

In a study by Li et al graphene oxide in hydrogel was used as a nano-carrier in order to increase the permeability and retention of cyclosporine in tissue. Efficacy evaluation was performed ex vivo on goatskin and modified Franz cells (diffusion area $1 \mathrm{~cm}^{2}$ ) and in vivo on rabbits for evaluation of irritant effect and on a TPA model in mice. The results demonstrated an increase in the permeability and tissue retention of cyclosporine while avoiding the effects of systemic administration (19) (Table II).

Mometasone furoate, a widely used corticosteroid in dermatological pathology with a certain anti-psoriasis effect, has recently been conditioned as an apasomal gel used topically against psoriasis. In a study by Shinde et al compared to the available cream that has a 5-h release, the release of the new formula took place over $24 \mathrm{~h}$ (20) (Table II).

Short-chain fatty acids in the colon induce regulatory $\mathrm{T}$ cell (Treg) generation. Schwarz and colleagues studied the effect of topically applied sodium butyrate using the IMQ model. The results revealed a IL-17 decrease and IL-10 and Foxp3 increased transcription. The data thus demonstrated that a Treg imbalance occurs in psoriasis that can be ameliorated by sodium butyrate (21) (Table II).

In a study by Guo et al salvianolic acid B with antiinflammatory, antioxidant and antitumoral properties was investigated using the IMQ model for psoriasis. Salvianolic acid B in microemulsion was shown to reduce acanthosis, epidermal proliferation, inhibit cytokine IL-23/IL-17 axis and increase skin hydration, having anti-psoriasis potential (22) (Table II).

Small interfering RNAs (siRNAs) can be used to suppress gene-specific alleles for various diseases such as psoriasis, lupus, hyperhidrosis, and neoplasms. For this purpose, the topical administration in the form of ionic liquids capable of forming non-covalent bonds with siRNA and 


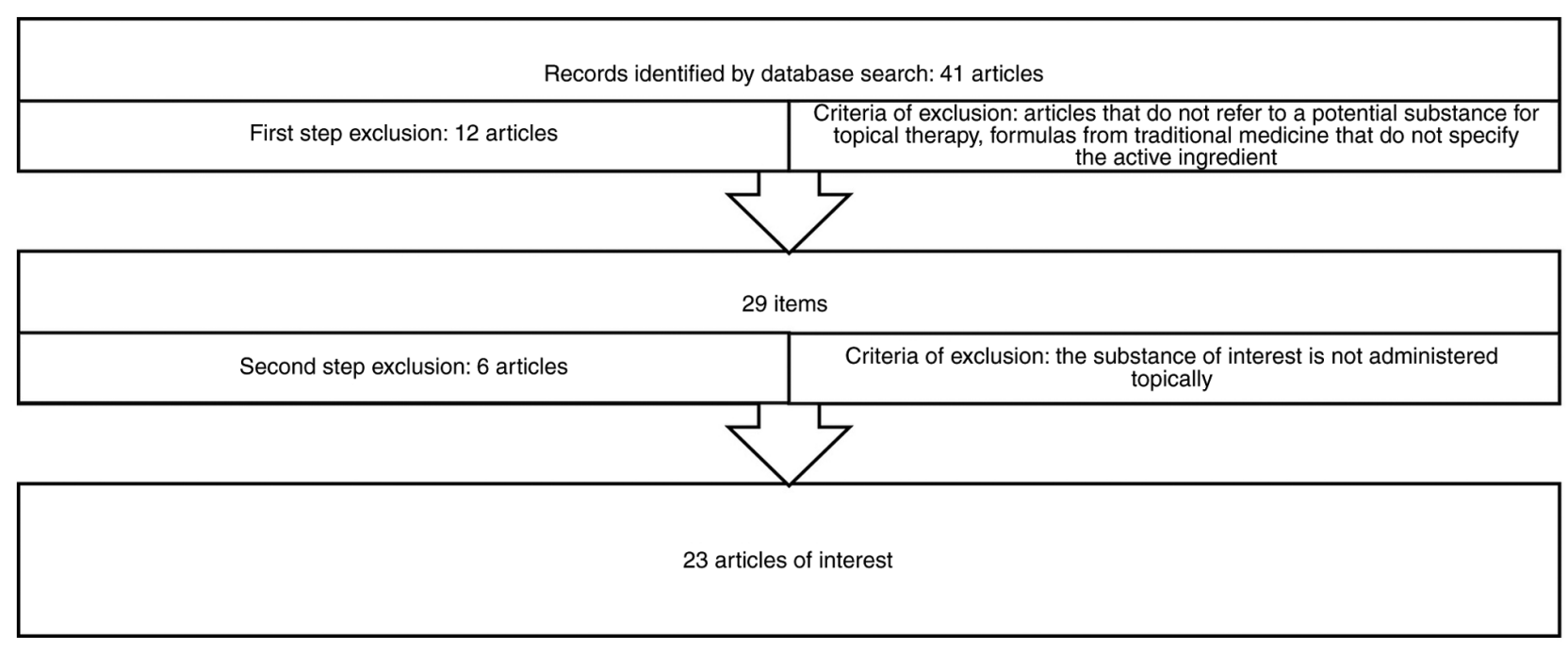

Figure 2. Criteria of inclusion/exclusion used in regards to the literature studies.

achieving adequate absorption was attempted in a study by Dharamdasani et al Hydrophobic cations were used to bind RNA and ionic liquid of choline-geranic acid to increase permeability. The experiments were performed in vitro on pig skin and in vivo on SKH-1E mice (23) (Table II).

Phosphodiesterase, an intracellular enzyme that hydrolyzes cGMP, is involved in multiple physiological processes. DC591017, a PDE4 (phosphodiesterase 4) inhibitor, was studied by Li et al in vivo and in vitro for its anti-psoriasis effect, and was found to decrease the inflammatory infiltrate and epidermal thickness. The IMQ and carrageenan models were used for this purpose (24) (Table II).

In a study by Di Fusco et al albendazole, an anthelmintic drug, was investigated in topical administrations using the experimental model of imiquimod in mice, with decreased keratinocyte proliferation, reduced keratin (K)6/K16 expression, reversibly inhibiting cell cycle by S-phase cell accumulation. Using this model, albendazole was shown to inhibit the protein kinase receptor (PKR), increase eukaryotic initiation factor 2 (eIF2 $\alpha$ ) phosphorylation and reduce CDC25A expression thus demonstrating an anti-psoriasis effect (25) (Table II).

Calcipotriol (CPT), a first-line topical drug in psoriasis vulgaris, was tested as an improved formula in regards to transdermal administration, avoiding adverse skin reactions due to loss of hydration in the epidermis. Bacillus amyloliquefaciens (named ZWJ strain) was identified as a producer of high- emulsification exopolysaccharides (EPS). The effect of the formula was tested by animal experiments, providing a scientific basis for future usage (26) (Table II).

In a study by Lee et al $\mathrm{CO}_{2}$ fractional laser was used to increase the permeability of the substance in the psoriatic plaque. A $64 \%$ decrease in IL-6 expression in the psoriatic plaque was demonstrated with topical application of IL-6 siRNA using the imiquimod psoriasis induction model. The conventional route of siRNA delivery for dermatological treatment is injection, with adverse reactions such as pain and difficult administration (27) (Table II).

After Feng et al (28) demonstrated in a previous study the involvement of microRNAs (miRNAs/miRs) in the pathogenesis of psoriasis, an anti-psoriasis effect was obtained after topical administration of antisense miRNA-210 in a reconstituted high-density lipoprotein nano-transporter gel. The formula decreased the level of miRNA-210 with reduction of erythema, scales, acanthosis, dermal infiltrate and of IL-17A, using the IMQ model (28) (Table II).

Thymoquinone, a liposoluble benzoquinone is the major substance in the volatile oil of Nigella sativa and has an anti-psoriasis effect. However, being practically insoluble in water and photosensitive, topical application in conventional formulas does not provide benefits. Thus, research using thymoquinone-loaded ethosomal vesicles in hydrogels was tested using the tail model for psoriasis. The study showed promising results in terms of anti-psoriasis effect of thymoquinone (29) (Table II).

Sea buckthorn oil extracted from Hippophae rhamnoides, which contains 16 types of monounsaturated and polyunsaturated fatty acids, was tested for its anti-psoriasis effect in vitro using human THP-1 cells, in vivo in systemic and topical administration in the model of paw edema in mice with carrageenan and on a CD-1 model of psoriasis in mice with TPA. The results of these studies were the reduction of reactive nitrogen species and $\mathrm{NF}-\kappa \mathrm{B}$ expression, depending on the concentration of the substance under investigation, along with pro-inflammatory cytokines IL-1 $\beta$, IL-6, inflammation and epidermal thickness reduction (30) (Table II).

Nitidine is a natural alkaloid extracted from Zanthoxylum nitidum (Roxb), with anti-proliferative properties using the ERK signaling pathway with antitumor potential in colorectal cancer (31) and a potent inhibitor of HaCaT keratinocyte proliferation in vitro, with decreased DNA synthesis, decrease in Ki67, cyclin A and D1 levels and with p53 protein increase. On experimental models with imiquimod/TPA topical application, epidermal thickness, edema and pro-inflammatory cytokine levels were decreased (32) (Table II).

Indole-3-carbinol (I3C) is a natural compound with anti-neoplastic effect. [1-(4-Chloro-3-nitrobenzenesulfonyl)1H-indol-3-yl]-methanol is a new I3C derivative. Upon topical application in the IMQ model, the latter compound decreased hyperplasia and inflammation, with suppression 
Table I. New substances and improved formulas in topical psoriasis treatment.

New topical substances tested

Substances in superior topical formulas/new mechanisms

Lycopene

Sodium butyrate

Salvianolic acid B

Small interfering RNAs (siRNAs)

in ionic liquids

Albendazole

Phosphodiesterase 4 inhibitors

Biomimetic reconstituted high-density

lipoprotein (rHDL) nanocarrier gel

containing miR-210 antisense

(NG-anti-miR-210)

Timoquinone in ethosomal vesicle

Sea buckthorn oil

(Hippophae rhamnoides)

Nitidine chloride

Melissa officinalis spp. Altissima extract

[1-(4-Chloro-3-nitrobenzenesulfonyl)-1H-

indol-3-yl]-methanol (CIM)

Manganese porphyrin
Cyclosporine-loaded Pluronic ${ }^{\circledR}$ F127 stabilized reduced graphene oxide hydrogel

Mometasone furoate-loaded aspasomal gel

Exopolysaccharides/calcipotriol emulsion

IL-6 siRNA-fractional $\mathrm{CO}_{2}$ laser

Topical gel of methotrexate $(0.25 \%)$ incorporated ethosomes and salicylic acid (2\%)

Topical methotrexate-entrapped deformable liposome

p38 protein kinase as a target with BIRB796 as inhibitor

Tazarotene enriched with $1 \%$ cineole

Celastrole in niosome gel

C16 with antagonistic effect against integrin $\alpha 5 \beta 1$ of cytokines specific to the MAPK, NF- $\kappa \mathrm{B}$ and AP-1 pathways (33) (Table II).

In a study by Chandra et al the combination of methotrexate with salicylic acid in ethosomal gel with a particle size of $376.04 \pm 3.47 \mathrm{~nm}$ was tested in the IMQ model and then compared to methotrexate solution. The results showed a $43 \%$ retention study for the gel compared to $13 \%$ for the solution, with decreased PASI and normalization of psoriatic plaques (34) (Table II).

In a study by Dimitris et al Melissa officinalis spp. Altissima extract (lemon balm) was tested using the IMQ model. Chemical analysis was performed to detect active metabolites. From the dichloromethane extract, seven triterpenes were isolated, i.e. ursolic acid, $2 \alpha$-hydroxy-ursolic acid, pomolic acid, $3 \beta$-stearyloxy-urs-12-ene, oleanolic acid, noropacursane and campesterol. The methanol extract yielded two phenolic acids (rosmarinic acid and methyl rosmarinate), which seem to be the major compounds of the total methanol extract. In addition, from the decoction, three phenolic acids were isolated: Caffeic acid, 3-(3,4-dihydroxyphenyl) lactic acid and rosmarinic acid. Extracts with dichloromethane and methanol and decoction were used. Triterpene derivatives of the dichloromethane extract were shown to be responsible for the anti-psoriasis effect. The polyphenols in the decoction were responsible for the strong antioxidant effect, with the strongest anti-psoriasis effect (35) (Table II).

Topical methotrexate embedded in deformable liposomes with phosphatidylcholine and oleic acid in concentrations of 0.05 and $0.1 \%$ demonstrated significant anti-psoriasis effect in the model of psoriasis with IMQ (36), without significant toxicity as in systemically administrated methotrexate (37-39). Methotrexate has low skin permeability due to high molecular weight and hydrophobicity, thus the liposomal form is optimal for increasing bioavailability (Table II).

The involvement of p38 protein kinase in the pathogenesis of psoriasis was investigated using the IMQ model. Anisomycin, a murine p38 activator with psoriasis-producing effect and BIRB796, a p38 inhibitor with anti-psoriasis effect, were used (40) (Table II).

Tazarotene is a synthetic topical retinoid used in the treatment of psoriasis but with a lipophilic structure causing irritating side effects. Recent in vitro and ex vivo research conducted by Elmowafy et al (41) regarding nanovesicles enriched with $1 \%$ cineole recorded a significant loading of tazarotene with a total of $81.51 \%$ in all epidermal layers. Statistically significant results were obtained in terms of PASI score, dermoscopic appearance, anti-psoriasis effect compared to the commercial reference product used (41).

MnTE-2-PyP (BMX-010) (manganese porphyrin) in topical applications is a new anti-psoriatic agent with antipruritic effect in nonspecific idiopathic prurigo and atopic dermatitis. Phase I study in 64 patients did not show any significant adverse reactions. The only toxic adverse reaction to maximum concentration in mice was reversible hypertension (42).

Celastrole, a triterpenoid extracted from Tripterygium, was developed in a new therapeutic formula, respectively in 147-nm niosomes. Results revealed erythema and scaly relief in the IMQ psoriasis model, with decreased IL-22, IL-23 and IL-17 levels, with an in vivo demonstrated increase in skin absorption (43) (Table II).

Integrin $\alpha 5 \beta 1$, as a fibronectin receptor, is expressed in excess in the non-lesional epidermis of psoriasis. In order to investigate whether this integrin is a potential target in psoriasis treatment, an antagonist ligand peptide called C16, was 


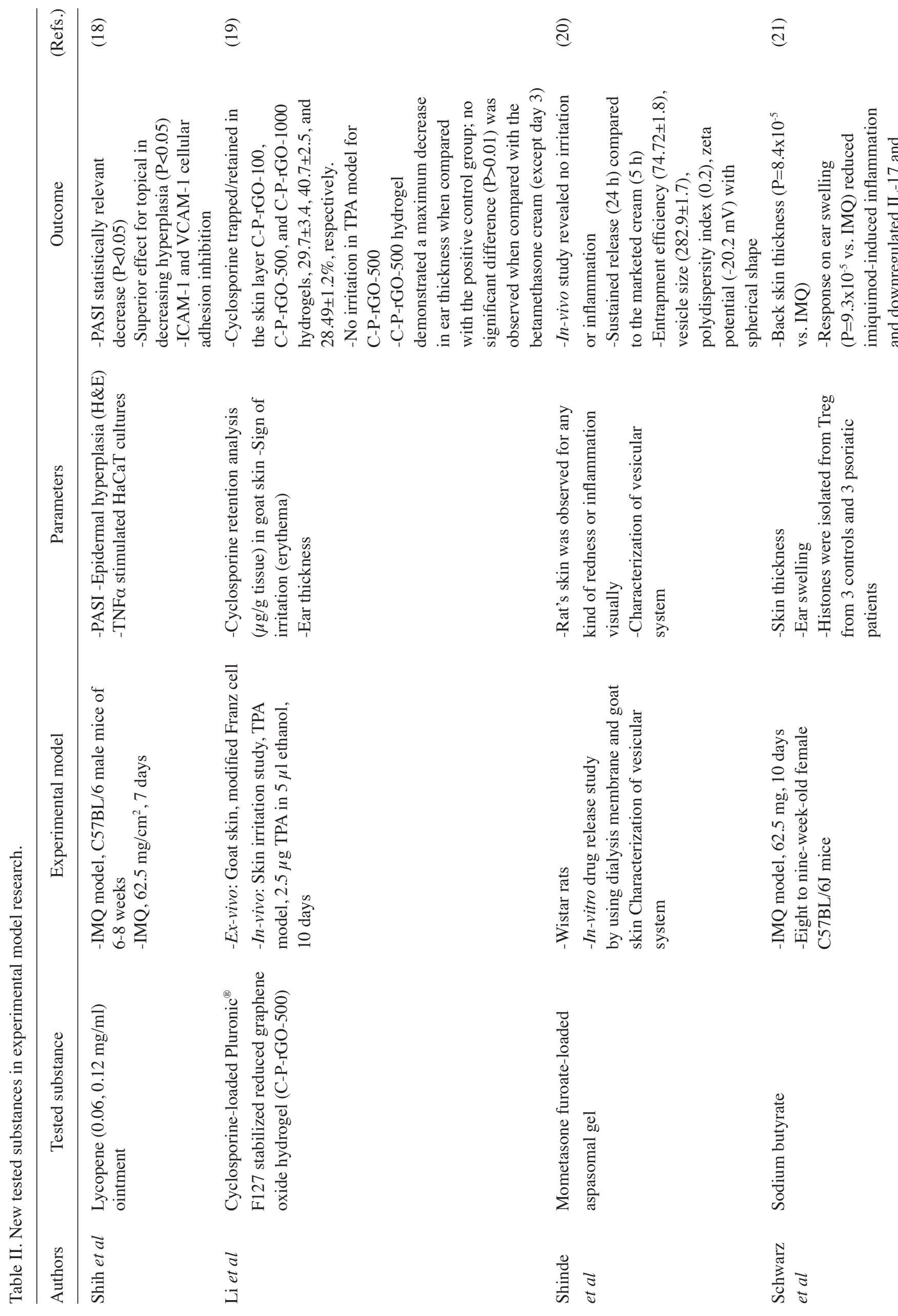




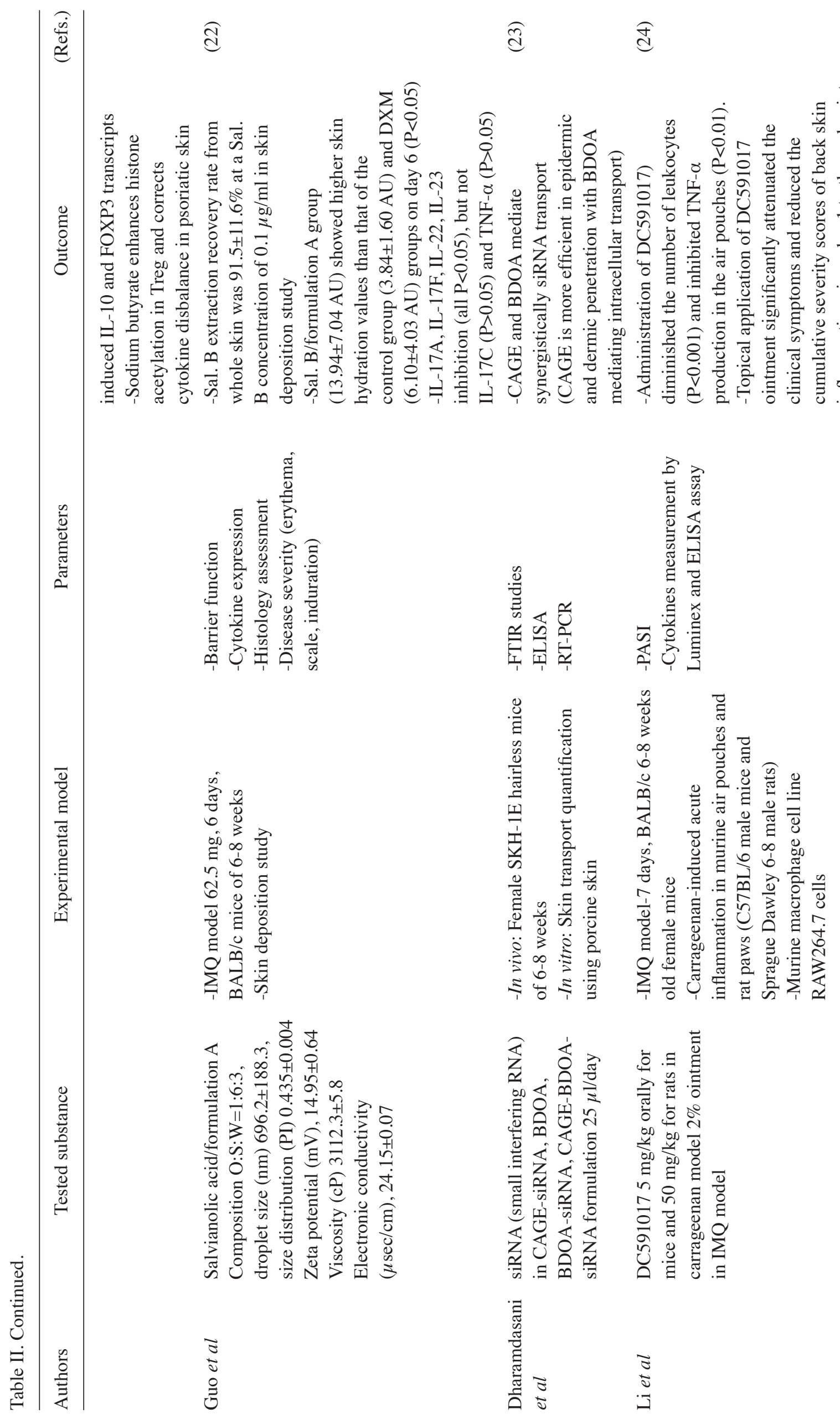




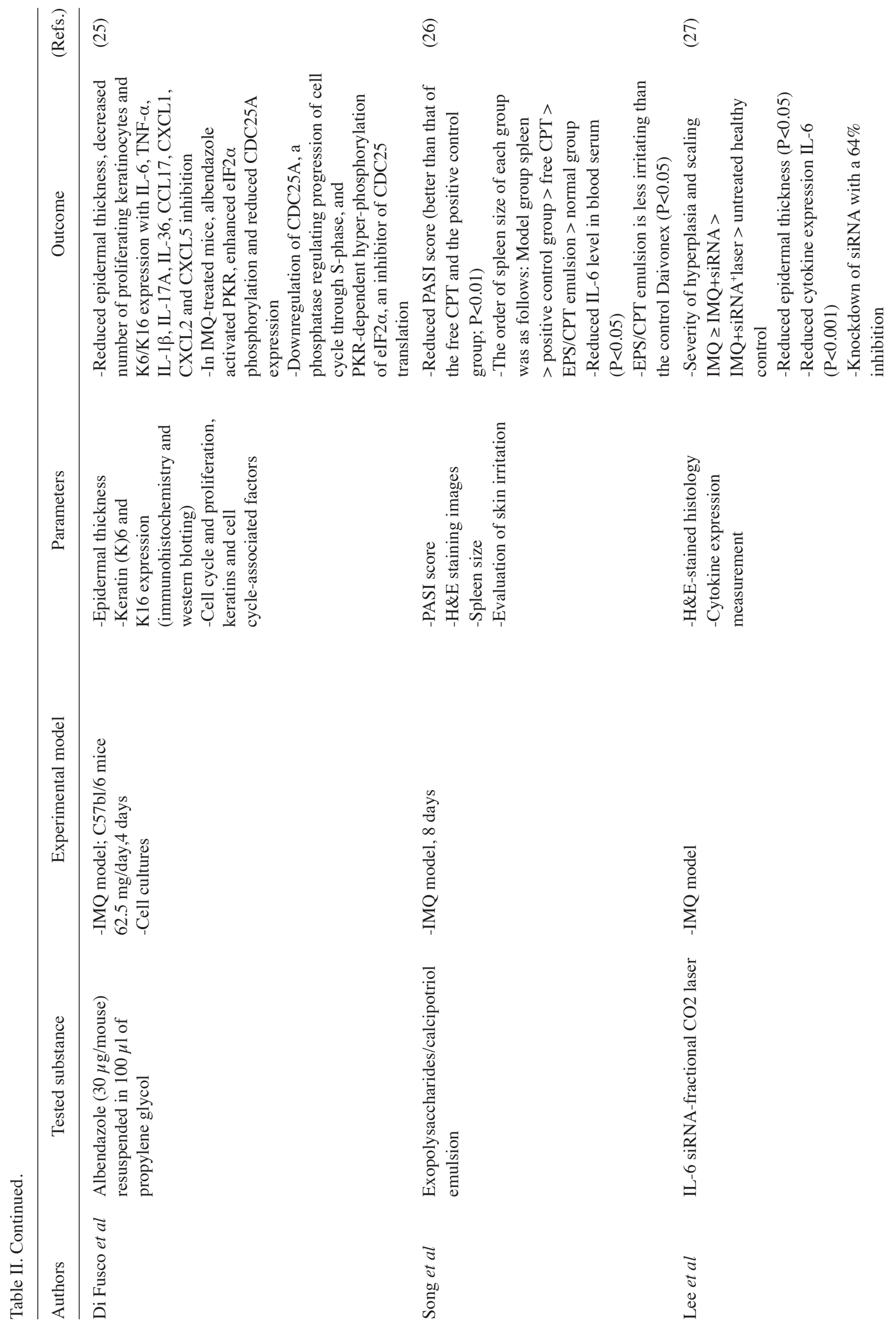




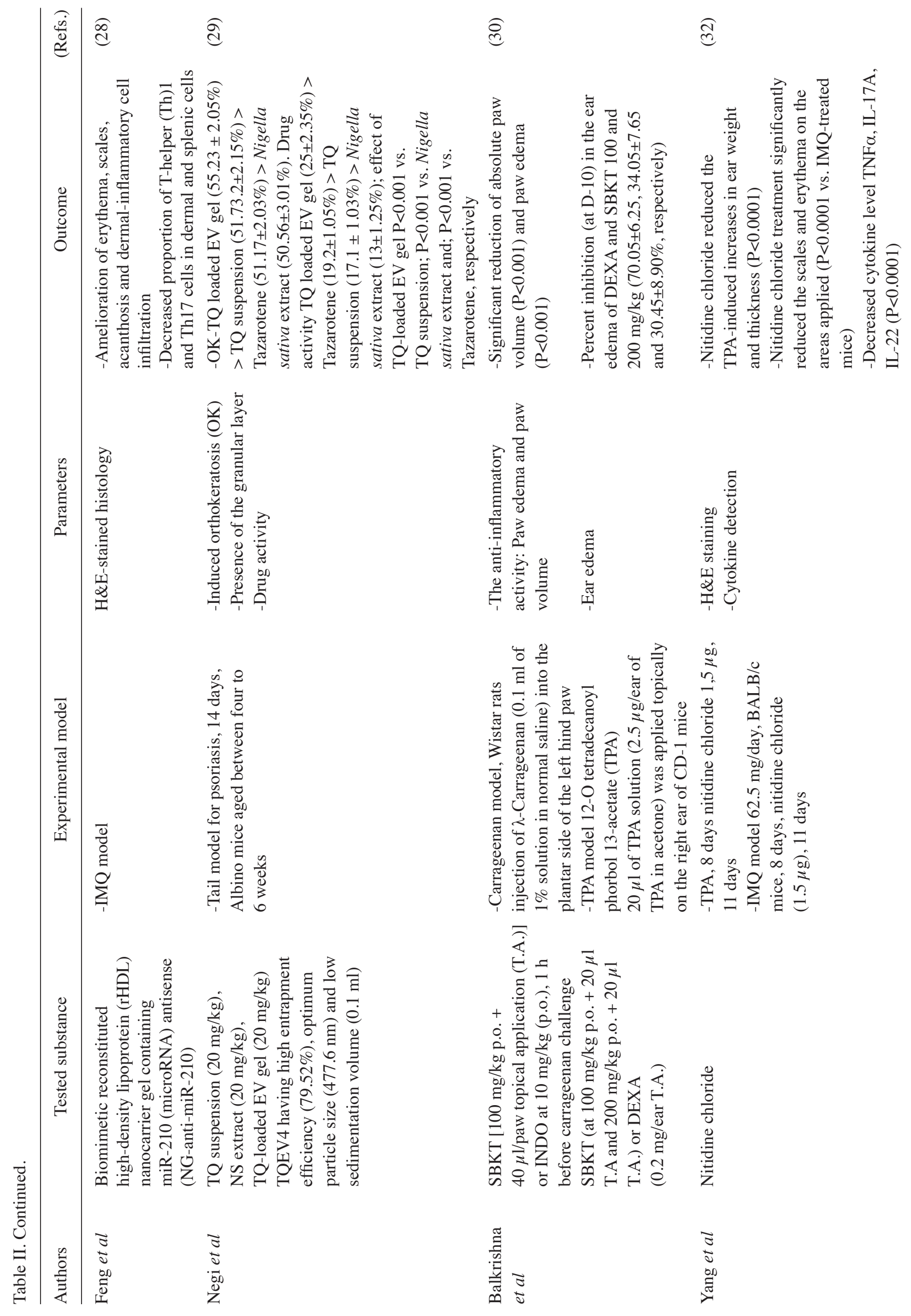




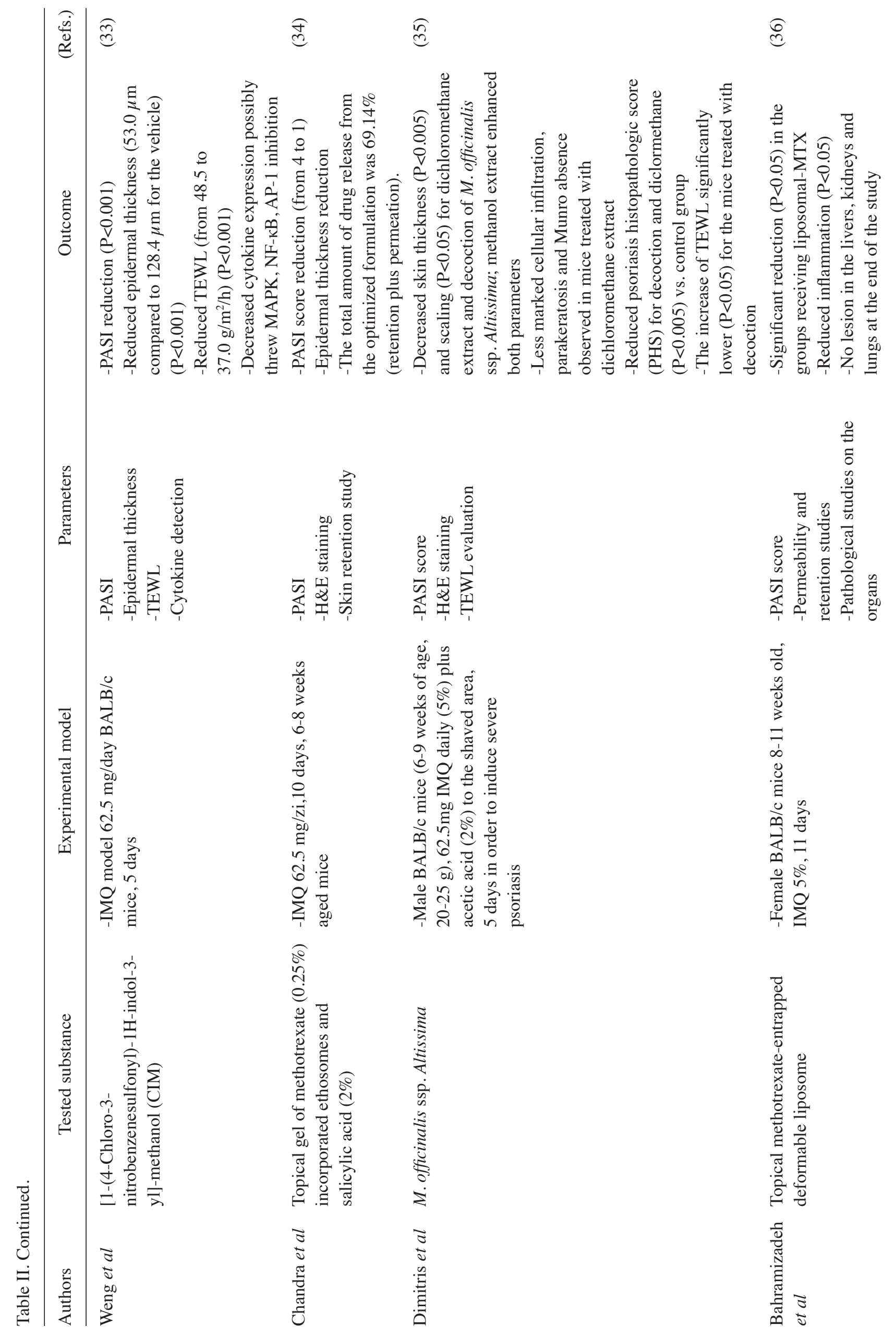




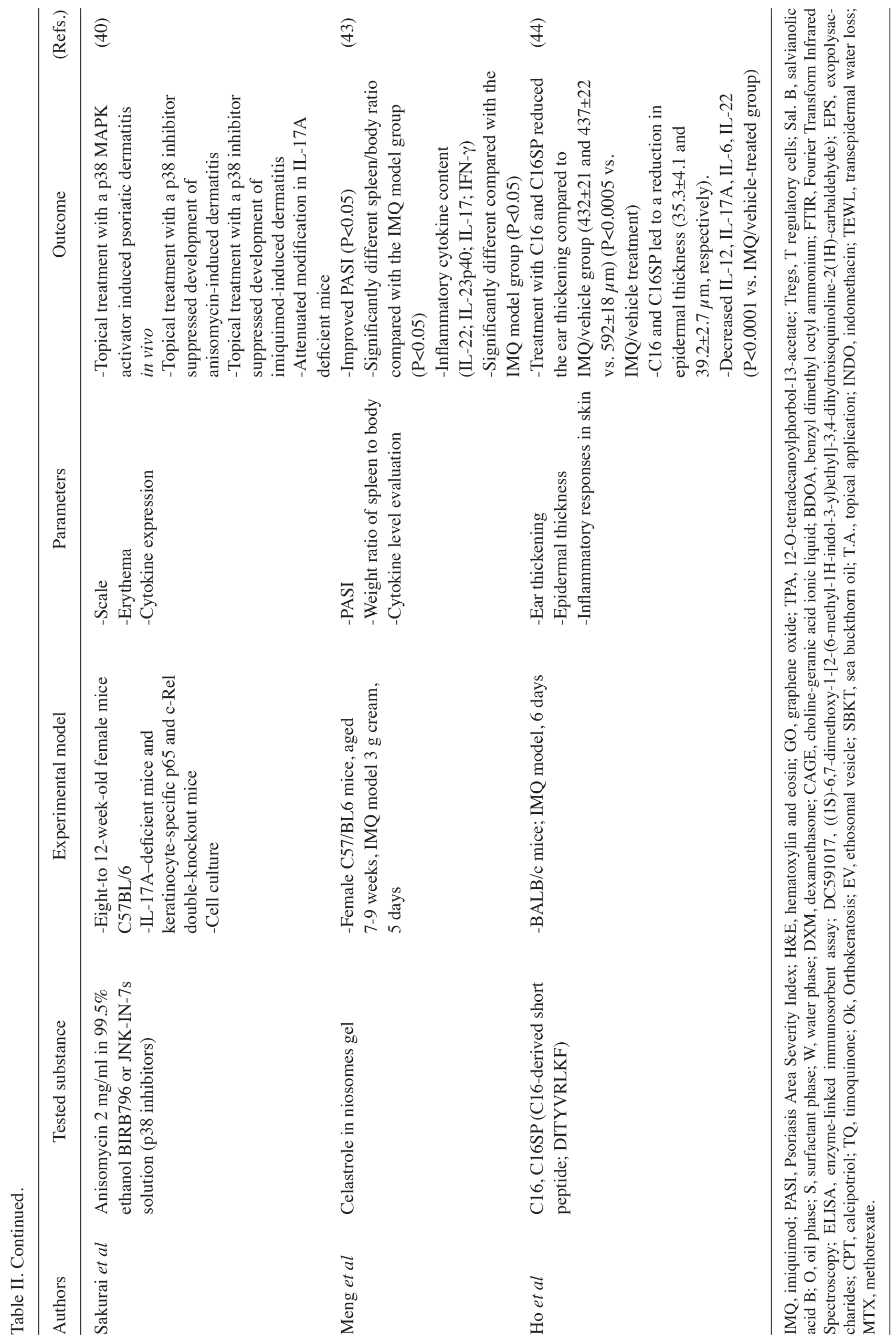


used. Using the IMQ model, Ho et al showed that topically administered $\mathrm{C} 16$ reduced epidermal hyperplasia, neutrophilic infiltrate and expression of pro-inflammatory mediators. Thus, $\alpha 5 \beta 1$ integrin is a potential target for anti-psoriasis therapy (44) (Table II).

\section{Discussion}

After studying the scientific literature from the last current year regarding the topical therapy of psoriasis, it was observed that the subject is of great interest, with multiple experimental research studies of new therapies or mechanisms of action of already known substances included in superior formulas, with increased skin absorption and penetrability.

Imiquimod (IMQ) can aggravate and even trigger psoriasis both at the site of application and remotely. The application of imiquimod to laboratory mice causes IL-23/IL-17 axisdependent changes, with influx of immune cells and cytokines such as IL-1 $\alpha$, IFN- $\alpha$, IL-23 and IL-6. On the other hand, the application of IMQ causes, possibly through a cAMPdependent mechanism, epidermal hyperplasia, thus resulting in phenotypic and histological changes of psoriasis $(45,46)$. This model was initially reported in 2009 and has been used most frequently in anti-psoriasis therapy research ever since. It consists of the topical application of IMQ to the ear and back of the laboratory mouse on day 0 of the experiment demonstrating the characteristic changes of psoriasis: Erythema, scale, and altered keratinocyte differentiation. The best results have been obtained by using B6/C57BL mice (47).

The current trend regarding the therapy of psoriasis is to use vehicles such as vesicular systems (liposomes and ethosomes). Liposomes are artificial spherical vesicles composed of cholesterol and natural phospholipids. They are hydrophobic and hydrophilic, biocompatible, non-immunogenic and increase the therapeutic index and efficacy of the active substance. The properties of liposomes differ depending on composition, size (from 0.025 to $2.5 \mu \mathrm{m}$ ) and preparation methods (48).

Liposomes are used with promising results for intracellular release systems: Antisense molecules, ribosomes, proteins, and DNA (48). Ethosomes are phospholipid elastic nanovesicles with a high ethanol content (20-45\%). Ethanol increases permeability and by interacting with the lipid polar end decreases the melting point of lipids in the stratum corneum, thus increasing cellular fluidity and permeability. Ethosomal systems are much more efficient in delivering the substance in terms of quantity and depth than liposomes and hydroalcoholic solutions (49). Niosomes are vesicles with non-ionic surfactant, lamellar structures formed by the combination of alkyl-polyglycerol surfactant with cholesterol. The concentration of cholesterol in liposomes is higher than that in niosomes so that the binding efficiency of the active substance is lower in the case of liposomes, which are less stable but also more expensive (50).

There were additional articles that did not meet the chosen criteria but were significant in regards to novel methods in psoriasis therapy.

Stress as an aggravating factor of psoriasis was analyzed using the imiquimod model in laboratory mice using a bottle emptying as a stressor stimulation. Thus, it was shown that stress worsened and prolonged imiquimod-induced psoriasis dermatitis, with increased levels of neurotransmitters such as substance P and IL-1 $\beta$ and IL-23p40 upregulation (51).

Estrogen receptors have been studied in relation to the development of psoriatic dermatitis in the IMQ model in laboratory mice. In one study, from day 2 of IMQ application, estrogenic agonists were administered orally: Either ER $\alpha$ selective agonist [propylpyrazoletriol (PPT) $2.5 \mathrm{mg} / \mathrm{kg}$ ] or $\mathrm{ER} \beta$ selective agonist [diarylpropionitrile (DPN) $2.5 \mathrm{mg} / \mathrm{kg}$ ]. Administration of PPT induced pruritic behavior and proinflammatory response, increasing the levels of IL-17 and IL-22, while DPN did not influence these aspects in any way. In addition, PPT also increased IL-23 levels by stimulating dendritic cells. This research concluded that the stimulation of $\alpha$ and not $\beta$ estrogen receptors is associated with pruritic and proinflammatory behavior in the IMQ model (52).

The IMQ psoriasis induction model was used to study the mechanism of TNF- $\alpha$ induction by FGF-7, using a specific anti-FGF-7 antibody called F-9 which decreased inflammation and PASI. It has thus been shown that a potential therapeutic target in anti-psoriasis therapy is the FGF-7 pathway (53). Recent research by Borek et al (54) showed that TGF- $\beta$-dependent factors in psoriasis plaque act mainly through the bone morphogenic protein (BMP) cascade with the influence of Langerhans cell subtype, promoting the classic changes in psoriasis. In vivo experimental models with Junf/fJunBf/fK5cre-ERT mice were used for this purpose. Thus, it can be concluded that by using the BMP pathway as a therapeutic target, the mechanism of psoriasis production can be suppressed (54).

Microglial healing peptide-1 (MHP1-AcN) inhibits Tolllike receptor (TLR)-related inflammation by RANK/RANK-L signaling in microglia and macrophages without activating osteoclasts. Inhibition of TLR is a feasible treatment strategy for psoriasis. Using the IMQ model, the effect of this substance was studied. The results were more than promising. In addition to a reduction in erythema and scales, there were significant decreases in IL-6, IL-23, and IL-17A (55).

Kaempferol, a natural flavonoid, was found to attenuate inflammation in the imiquimod model, decreasing the proinflammatory cytokines IL-6, IL-17-A, TNF- $\alpha$, inhibiting the $\mathrm{NF}-\kappa \mathrm{B}$ pathway, suppressing $\mathrm{T}$ lymphocytes in vitro and demonstrating anti-psoriasis potential (56).

\section{Conclusions}

Recent studies in topical psoriasis underline the importance of animal experimental research in dermatology, providing a starting point for developing new therapeutic approach in one of the most frequently diagnosed chronic diseases. Two trends can be found from the review. One trend is represented by the discovery of new topical active substances with potential anti-psoriasis effect: Lycopene, sodium butyrate, salvianolic acid $\mathrm{B}$, albendazole, phosphodiesterase 4 inhibitors, thymoquinone, and nitidine chloride. Another trend is represented by the research of new mechanisms of action for some active substances, already used in the topical therapy of psoriasis but in superior topical formulations in order to increase their topical biodisponibility. Vesicular systems are now providing the best vehicle for topical therapy, thus easing the action of the active substances at the target site. 


\section{Acknowledgements}

Not applicable.

\section{Funding}

No funding was received.

\section{Availability of data and materials}

Not applicable.

\section{Authors' contributions}

DAMN analyzed the data from the literature regarding substances and their mechanism of action. MN analyzed the results. AM designed the final aspect of the manuscript. MC analyzed the clinical implications of the new formulas researched. OAC analyzed all of the data and wrote the conclusions. All the authors critically revised the manuscript and read and approved its final version.

\section{Ethics approval and consent to participate}

Not applicable.

\section{Patient consent for publication}

Not applicable.

\section{Competing interests}

The authors declare that they have no competing interests.

\section{References}

1. Bologna JL, Schaffer JV and Cerroni L: Dermatology. 4th edition. Elsevier, Amsterdam, pp138-159, 2018.

2. Caruntu C, Boda D, Dumitrascu G, Constantin C and Neagu M: Proteomics focusing on immune markers in psoriatic arthritis. Biomark Med 9: 513-528, 2015.

3. Rendon A and Schäkel K: Psoriasis pathogenesis and treatment. Int J Mol Sci 20: 1475, 2019.

4. Alecu M, Coman G, Mușetescu, Cojoacă ME and Coman OA: Dermatology facing autoinflammatory syndrome. Rom J Morphol Embryol 56: 7-14, 2015.

5. Costescu M, Coman OA, Tampa M, Tudose I, Coman L and Georgescu SR: Axillary basal cell carcinoma-a rare form of a frequent kind of carcinoma. Rom J Morphol Embryol 54 (Suppl 3): S851-S856, 2013.

6. Nicolae I, Nicolae CD, Coman OA, Stefanescu M, Coman L and Ardeleanu C: Serum total gangliosides level: Clinical prognostic implication. Rom J Morphol Embryol 52: 1277-1281, 2011.

7. Algahtani M, Ahmad M, Nourein I and Ahmad J: Co-delivery of imiquimod and curcumin by nanoemugel for improved topical delivery and reduced psoriasis-like skin lesions. Biomolecules 10: 968, 2020.

8. Evans EA, Sayers SR, Kodji X, Xia Y, Shaikh M, Rizvi A, Frame J, Brain SD, Philpott MP, Hannen RF and Caton PW: Psoriatic skin inflammation induces a pre-diabetic phenotype via the endocrine actions of skin secretome. Mol Metab 41: 101047, 2020.

9. Xu Z, Qin Z, Zhang J and Wang Y: Microglia-mediated chronic psoriatic itch induced by imiquimod. Mol Pain: Jun 24, 2020 (Epub ahead of print). doi: 10.1177/1744806920934998.

10. Bai X, Yu C, Yang L, Luo Y, Zhi D, Wang G and Dang E: Anti-psoriatic properties of paeoniflorin: Suppression of the NF-kappaB pathway and Keratin 17. Eur J Dermatol 30: 243-250, 2020.
11. Ren F, Zhang M, Zhang $\mathrm{C}$ and Sang H: Psoriasis-like inflammation induced renal dysfunction through the TLR/NF- $\mathrm{B}$ signal pathway. Biomed Res Int 2020: 3535264, 2020.

12. Duan X, Liu X, Liu N, Huang Y, Jin Z, Zhang S, Ming Z and Chen $\mathrm{H}$ : Inhibition of keratinocyte necroptosis mediated by RIPK1/RIPK3/MLKL provides a protective effect against psoriatic inflammation. Cell Death Dis 11: 134, 2020.

13. Yu S, Wu X, Shi Z, Huynh M, Jena PK, Sheng L, Zhou Y, Han D, Wan YY and Hwang ST: Diet-induced obesity exacerbates imiquimod-mediated psoriasiform dermatitis in anti-PD-1 antibody-treated mice: Implications for patients being treated with checkpoint inhibitors for cancer. J Dermatol Sci 97: 194-200, 2020.

14. Man MQ and Elias PM: Could inflammaging and its sequelae be prevented or mitigated? Clin Interv Aging 14: 2301-2304, 2019.

15. Shankar GM, Alex VV, Nisthul AA, Bava SV, Sundaram S, Retnakumari AP, Chittalakkottu S and Anto RJ: Pre-clinical evidences for the efficacy of tryptanthrin as a potent suppressor of skin cancer. Cell Prolif 53: e12710, 2020.

16. Chiang CC, Cheng WJ, Lin CY, Lai KH, Ju SC, Lee C, Yang SH and Hwang TL: Kan-Lu-Hsiao-Tu-Tan, a traditional Chinese medicine formula, inhibits human neutrophil activation and ameliorates imiquimod-induced psoriasis-like skin inflammation. J Ethnopharmacol 246: 112246, 2020.

17. Rapalli VK, Singhvi G, Gorantla S, Waghule T, Dubey SK, Saha RN, Hasnain MS and Nayak AK: Stability indicating liquid chromatographic method for simultaneous quantification of betamethasone valerate and tazarotene in in vitro and ex vivo studies of complex nanoformulation. J Sep Sci 42: 3413-3420, 2019.

18. Shih CM, Hsieh CK, Huang CY, Huang CY, Wang KH, Fong TH, Trang NT, Liu KT and Lee AW: Lycopene inhibit IMQ-induced psoriasis-like inflammation by inhibiting ICAM-1 production in mice. Polymers (Basel) 12: 1521, 2020.

19. Li Q, Li F, Qi X, Wei F, Chen H and Wang T: Pluronic ${ }^{\circledR}$ F127 stabilized reduced graphene oxide hydrogel for the treatment of psoriasis: In vitro and in vivo studies. Colloids Surf B Biointerfaces 195: 111246, 2020.

20. Shinde G, Desai P, Shelke S, Patel R, Bangale G and Kulkarni D: Mometasone furoate-loaded aspasomal gel for topical treatment of psoriasis: Formulation, optimization, in vitro and in vivo performance. J Dermatolog Treat: Jul 8, 2020 (Epub ahead of print). doi: 10.1080/09546634.2020.1789043.

21. Schwarz A, Philippsen R and Schwarz T: Induction of regulatory T cells and correction of cytokine dysbalance by short-chain fatty acids: Implications for psoriasis therapy. J Invest Dermatol 141: 95-104.e2, 2021

22. Guo JW, Cheng YP, Liu CY, Thong HY, Huang CJ, Lo Y, Wu CY and Jee SH: Salvianolic acid B in microemulsion formulation provided sufficient hydration for dry skin and ameliorated the severity of imiquimod-induced psoriasis-like dermatitis in mice. Pharmaceutics 12: 457, 2020.

23. Dharamdasani V, Mandal A, Qi QM, Suzuki I, Bentley MV and Mitragotri S: Topical delivery of siRNA into skin using ionic liquids. J Control Release 323: 475-482, 2020.

24. Li H, Li J, Zhang X, Feng C, Fan C, Yang X, Zhang R, Zhu F, Zhou Y, Xu Y, et al: DC591017, a phosphodiesterase-4 (PDE4) inhibitor with robust anti-inflammation through regulating PKA-CREB signaling. Biochem Pharmacol 177: 113958, 2020.

25. Di Fusco D, Stolfi C, Di Grazia A, Dinallo V, Laudisi F, Marafini I, Colantoni A, Monteleone I and Monteleone G: Albendazole negatively regulates keratinocyte proliferation. Clin Sci (Lond) 134: 907-920, 2020.

26. Song B, Song R, Cheng M, Chu H, Yan F and Wang Y: Preparation of calcipotriol emulsion using bacterial exopolysaccharides as emulsifier for percutaneous treatment of psoriasis vulgaris. Int J Mol Sci 21: 77, 2020.

27. Lee WR, Lin YK, Alalaiwe A, Wang PW, Liu PY and Fang JY: Fractional laser-mediated siRNA delivery for mitigating psoriasis-like lesions via IL-6 silencing. Mol Ther Nucleic Acid 19: 240-251, 2020.

28. Feng H, Wu R, Zhang S, Kong Y, Liu Z, Wu H, Wang H, Su Y, Zhao $\mathrm{M}$ and Lu Q: Topical administration of nanocarrier miRNA-210 antisense ameliorates imiquimod-induced psoriasislike dermatitis in mice. J Dermatol 47: 147-154, 2020.

29. Negi P, Sharma I, Hemrajani C, Rathore C, Bisht A, Raza K and Katare OP: Thymoquinone-loaded lipid vesicles: A promising nanomedicine for psoriasis. BMC Complement Altern Med 19: $334,2019$. 
30. Balkrishna A, Sakat SS, Joshi K, Joshi K, Sharma V, Ranjan R, Bhattacharya K and Varshney A: Cytokines driven anti-inflammatory and anti-psoriasis like efficacies of nutraceutical sea buckthorn (Hippophae rhamnoides) oil. Front Pharmacol 10: 1186, 2019.

31. Zhai H, Hu S, Liu T, Wang F, Wang X, Wu G, Zhang Y, Sui M, Liu $\mathrm{H}$ and Jiang L: Nitidine chloride inhibits proliferation and induces apoptosis in colorectal cancer cells by suppressing the ERK signaling pathway. Mol Med Rep 13: 2536-2542, 2016.

32. Yang XG, Jiang BW, Jing QQ, Li WJ, Tan LP, Bao YL, Song ZB, Yu CL, Liu L, Liu YC and Li YX: Nitidine chloride induces S phase cell cycle arrest and mitochondria-dependent apoptosis in $\mathrm{HaCaT}$ cells and ameliorates skin lesions in psoriasis-like mouse models. Eur J Pharmacol 863: 172680, 2019.

33. Weng JR, Huang TH, Lin ZC, Alalaiwe A and Fang JY: Cutaneous delivery of [1-(4-chloro-3-nitrobenzenesulfonyl)-1Hindol-3-yl]-methanol, an indole-3-carbinol derivative, mitigates psoriasiform lesion by blocking MAPK/NF- $\mathrm{KB} / \mathrm{AP}-1$ activation. Biomed Pharmacother 119: 109398, 2019.

34. Chandra A, Aggarwal G, Manchanda S and Narula A: Development of topical gel of methotrexate incorporated ethosomes and salicylic acid for the treatment of psoriasis. Pharm Nanotechnol 7: 362-374, 2019.

35. Dimitris D, Ekaterina-Michaela T, Christina K, Ioannis S, Ioanna SK, Aggeliki L, Sophia H, Michael R and Helen S: Melissa officinalis ssp. altissima extracts: A therapeutic approach targeting psoriasis in mice. J Ethnopharmacol 246: 112208, 2020.

36. Bahramizadeh M, Bahramizadeh M, Kiafar B, Jafarian AH, Nikpoor AR, Hatamipour M, Esmaily H, Rezaeemehr Z, Golmohammadzadeh S, Moosavian SA and Jafari MR: Development, characterization and evaluation of topical methotrexate-entrapped deformable liposome on imiquimod-induced psoriasis in a mouse model. Int J Pharm 569: 118623, 2019.

37. Negrei C, Ginghină O, Caruntu C, Burcea Dragomiroiu G, Jinescu $G$ and Boda D: Investigation relevance of methotrexate polyglutamates in biological systems by high performance liquid chromatography. Rev Chim Bucharest 66: 766-768, 2015.

38. Negrei C, Caruntu C, Ginghina O, Dragomiroiu GT, Toderescu CD and Boda D: Qualitative and quantitative determination of methotrexate polyglutamates in erythrocytes by high performance liquid chromatography. Rev Chim-Bucharest 66: 607-610, 2015.

39. Boda D, Negrei C, Nicolescu F and Badalau C: Assessment of some oxidative stress parameters in methotrexate treated psoriasis patients. Farmacia 62: 704-710, 2014.

40. Sakurai K, Dainichi T, Garcet S, Tsuchiya S, Yamamoto Y, Kitoh A, Honda T, Nomura T, Egawa G, Otsuka A, et al: Cutaneous p38 mitogen-activated protein kinase activation triggers psoriatic dermatitis. J Allergy Clin Immunol 144: 1036-1049, 2019.

41. Elmowafy E, El-Gogary RI, Ragai MH and Nasr M: Novel antipsoriatic fluidized spanlastic nanovesicles: In vitro physicochemical characterization, ex vivo cutaneous retention and exploratory clinical therapeutic efficacy. Int J Pharm 568: 118556, 2019.

42. Gad SC, Sullivan DW Jr, Mujer CV, Spainhour CB and Crapo JD: Nonclinical safety and toxicokinetics of MnTE-2-PyP (BMX-010), a topical agent in phase 2 trials for psoriasis and atopic dermatitis. Int J Toxicol 38: 291-302, 2019.
43. Meng S, Sun L, Wang L, Lin Z, Liu Z, Xi L, Wang Z and Zheng Y: Loading of water-insoluble celastrol into niosome hydrogels for improved topical permeation and anti-psoriasis activity. Colloids Surf B Biointerfaces 182: 110352, 2019.

44. Ho TC, Yeh SI, Chen SL and Tsao YP: The psoriasis therapeutic potential of a novel short laminin peptide C16. Int J Mol Sci 20: 3144, 2019.

45. Căruntu C, Boda D, Caruntu A, Rotaru M, Baderca F and Zurac S: In vivo imaging techniques for psoriatic lesions. Rom J Morphol Embryol 55 (Suppl 3): S1191-S1196, 2014.

46. Batani A, Branisteanu DE, Ilie MA, Boda D, Ianosi S, Ianosi G and Caruntu C: Assessment of dermal papillary and microvascular parameters in psoriasis vulgaris using in vivo reflectance confocal microscopy. Exp Ther Med 15: 1241-1246, 2018.

47. Swindell WR, Michaels KA, Sutter AJ, Diaconu D, Fritz Y, Xing X, Sarkar MK, Liang Y, Tsoi A, Gudjonsson JE and Ward NL: Imiquimod has strain-dependent effects in mice and does not uniquely model human psoriasis. Genome Med 9: 24 , 2017.

48. Akbarzadeh A, Rezaei-Sadabad R, Davaran S, Joo SW, Zargami N, Hanifehpour Y, Samiei M, Kouhi M and NejatiKoshki K: Liposome: Classification, preparation, and applications. Nanoscale Ress Lett 8: 102, 2013.

49. Verma P and Pathak K: Therapeutic and cosmeceutical potential of ethosomes: An overview. J Adv Pharm Technol Res 1: 274-282, 2010.

50. Kazi KM, Mandal AS, Biswas N, Guha A, Chatterjee S, Behera $\mathrm{M}$ and Kuotsu K: Niosome: A future of targeted drug delivery systems. J Adv Pharm Technol Res 1: 374-380, 2010.

51. Wang Y, Li P, Zhang L, Fu J, Di T, Li N, Meng Y, Guo J and Zhao J: Stress aggravates and prolongs imiquimod-induced psoriasis-like epidermal hyperplasia and IL-1//IL-23p40 production. J Leukoc Biol 108: 267-281, 2020

52. Iwano R, Iwashita N, Takagi Y and Fukuyama T: Estrogen receptor $\alpha$ activation aggravates imiquimod-induced psoriasislike dermatitis in mice by enhancing dendritic cell interleukin-23 secretion. J Appl Toxicol 40: 1353-1361, 2020.

53. Pu J, Wang R, Zhang $\mathrm{G}$ and Wang J: FGF-7 facilitates the process of psoriasis by inducing TNF- $\alpha$ expression in HaCaT cells. Acta Biochim Biophys Sin (Shanghai) 51: 1056-1063, 2019.

54. Borek I, Köffel R, Feichtinger J, Spies M, Glitzner-Zeis E, Hochgerner M, Sconocchia T, Krump C, Tam-Amersdorfer C, Passegger C, et al: BMP7 aberrantly induced in the psoriatic epidermis instructs inflammation-associated langerhans cells. J Allergy Clin Immunol 145: 1194-1207.e11, 2020.

55. Ju N, Shimamura M, Hayashi H, Ikeda Y, Yoshida S, Nakamura A, Morishita R, Rakugi $\mathrm{H}$ and Nakagami H: Preventative effects of the partial RANKL peptide MHP1-AcN in a mouse model of imiquimod-induced psoriasis. Scic Rep 9: 15434, 2019.

56. Liu C, Liu H, Lu C, Deng J, Yan Y, Chen H, Wang Y, Liang C, Wei J, Han L and Dai Z: Kaempferol attenuates imiquimodinduced psoriatic skin inflammation in a mouse model. Clin Exp Immunol 198: 403-415, 2019.

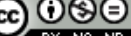

This work is licensed under a Creative Commons Attribution-NonCommercial-NoDerivatives 4.0 International (CC BY-NC-ND 4.0) License. 